\title{
Algorithm for Solving a New System of Generalized Variational Inclusions in Hilbert Spaces
}

\author{
Shamshad Husain and Sanjeev Gupta \\ Department of Applied Mathematics, Faculty of Engineering \& Technology, Aligarh Muslim University, Aligarh 202002, India \\ Correspondence should be addressed to Sanjeev Gupta; guptasanmp@gmail.com
}

Received 23 March 2013; Accepted 29 April 2013

Academic Editor: Raouf Boucekkine

Copyright (c) 2013 S. Husain and S. Gupta. This is an open access article distributed under the Creative Commons Attribution License, which permits unrestricted use, distribution, and reproduction in any medium, provided the original work is properly cited.

We introduce and study a new system of generalized variational inclusions involving $H(\cdot, \cdot)$-cocoercive and relaxed $(p, q)$-cocoercive operators, which contain the systems of variational inclusions and the systems of variational inequalities, variational inclusions, and variational inequalities as special cases. By using the resolvent technique for the $H(\cdot, \cdot)$-cocoercive operators, we prove the existence of solutions and the convergence of a new iterative algorithm for this system of variational inclusions in Hilbert spaces. An example is given to justify the main result. Our results can be viewed as a generalization of some known results in the literature.

\section{Introduction}

Variational inclusions have been widely studied in recent years. The theory of variational inclusions includes variational, quasi-variational, variational-like inequalities as special cases. Various kinds of iterative methods have been studied to solve the variational inclusions. Among these methods, the resolvent operator technique to study the variational inclusions has been widely used by many authors. For details, we refer to [1-15]. For applications of variational inclusions, see [16].

Fang and Huang, Lan, Cho, and Verma, and kazmi investigated several resolvent operators for generalized operators such as $H$-monotone, $A$-monotone, $H$-accretive, $A$-accretive, $(H, \eta)$-accretive, $(H, \eta)$-monotone, $(A, \eta)$-accretive, $M$-proximal, and $M-\eta$-proximal mappings. For further details, we refer to $[2-6,8-10,13]$ and the references therein. Very recently, Zou and Huang [15] introduced and studied $H(\cdot, \cdot)$ accretive operators, Xu and Wang [14] introduced and studied $(H(\cdot, \cdot), \eta)$-monotone operators, and Ahmad et al. [1] introduced and studied $H(\cdot, \cdot)$-cocoercive operators.

Inspired and motivated by researches going on in this area, we introduce and study a new system of generalized variational inclusions in Hilbert spaces. By using the resolvent operator technique for the $H(\cdot, \cdot)$-cocoercive operator, we develop a new class of iterative algorithms to solve a class of relaxed cocoercive variational inclusions associated with $H(\cdot, \cdot)$-cocoercive operators in Hilbert space. For illustration of Definitions 2, 5 and main result Theorem 19 Examples 4, 6, and 20 are given, respectively. Our results can be viewed as a refinement and improvement of Bai and Yang [2], Huang and Noor [17], and Noor et al. [11].

\section{Preliminaries}

Throughout this paper, we suppose that $X$ is a real Hilbert space endowed with a norm $\|\cdot\|$ and an inner product $\langle\cdot, \cdot\rangle$, respectively. $2^{X}$ is the family of all the nonempty subsets of $X$.

In the sequel, let us recall some concepts.

Definition 1 (see $[18,19])$. A mapping $g: X \rightarrow X$ is said to be

(i) $\lambda_{g}$-Lipschitz continuous if there exists a constant $\lambda_{g}^{g}>0$ such that

$$
\|g(x)-g(y)\| \leq \lambda_{g}\|x-y\|, \quad \forall x, y \in X .
$$

(ii) monotone if

$$
\langle g(x)-g(y), x-y\rangle \geq 0, \quad \forall x, y \in X .
$$


(iii) $\xi$-strongly monotone if there exists a constant $\xi>0$ such that

$$
\langle g(x)-g(y), x-y\rangle \geq \xi\|x-y\|^{2}, \quad \forall x, y \in X .
$$

(iv) $\alpha$-expansive if there exists a constant $\alpha>0$ such that

$$
\|g(x)-g(y)\| \geq \alpha\|x-y\|, \quad \forall x, y \in X .
$$

if $\alpha=1$, then it is expansive.

Definition 2 (see [1]). Let $H: X \times X \rightarrow X$ and $A, B: X \rightarrow X$ be the mappings.

(i) $H(A, \cdot)$ is said to be $\mu$-cocoercive with respect to $A$ if there exists a constant $\mu>0$ such that

$$
\begin{aligned}
& \langle H(A x, u)-H(A y, u), x-y\rangle \\
& \quad \geq \mu\|A x-A y\|^{2}, \quad \forall x, y \in X .
\end{aligned}
$$

(ii) $H(\cdot, B)$ is said to be $\gamma$-relaxed cocoercive with respect to $B$ if there exists a constant $\gamma>0$ such that

$$
\begin{aligned}
& \langle H(u, B x)-H(u, B y), x-y\rangle \\
& \quad \geq(-\gamma)\|B x-B y\|^{2}, \quad \forall x, y \in X .
\end{aligned}
$$

(iii) $H(A, \cdot)$ is said to be $\delta_{1}$-Lipschitz continuous with respect to $A$ if there exists a constant $\delta_{1}>0$ such that

$$
\|H(A x, \cdot)-H(A y, \cdot)\| \leq \delta_{1}\|x-y\|, \quad \forall x, y \in X .
$$

(iv) $H(\cdot, B)$ is said to be $\delta_{2}$-Lipschitz continuous with respect to $B$ if there exists a constant $\delta_{2}>0$ such that

$$
\|H(\cdot, B x)-H(\cdot, B y)\| \leq \delta_{2}\|x-y\|, \quad \forall x, y \in X .
$$

Definition 3. A multivalued mapping $M: X \rightarrow 2^{X}$ is said to be $\mu^{\prime}$-cocoercive if there exists a constant $\mu^{\prime}>0$ such that

$$
\begin{aligned}
& \langle u-v, x-y\rangle \\
& \geq \mu^{\prime}\|u-v\|^{2}, \quad \forall x, y \in H \text { for some } u \in M(x), v \in M(y) .
\end{aligned}
$$

Example 4 (see [1]). Let $X=\mathbb{R}^{2}$ with usual inner product. Let $A, B: \mathbb{R}^{2} \rightarrow \mathbb{R}^{2}$ be defined by

$$
\begin{array}{rr}
A x=\left(2 x_{1}-2 x_{2},-2 x_{1}+4 x_{2}\right), & B y=\left(y_{1}-y_{2},-y_{2}\right), \\
& \forall\left(x_{1}, x_{2}\right),\left(y_{1}, y_{2}\right) \in \mathbb{R}^{2},
\end{array}
$$

such that $\left(x_{1}, x_{2}\right),\left(y_{1}, y_{2}\right) \in \mathbb{R}^{2}$. Suppose that $H: \mathbb{R}^{2} \times \mathbb{R}^{2} \rightarrow$ $\mathbb{R}^{2}$ is defined by

$$
H(A x, B y)=A x+B y .
$$

Then $H(A, B)$ is $1 / 6$-cocoercive with respect to $A$ and $1 / 2$ relaxed cocoercive with respect to $B$.
Definition 5 (see [1]). Let $A, B: X \rightarrow X, H: X \times X \rightarrow$ $X$ be three single-valued mappings. Let $M: X \rightarrow 2^{X}$ be a set-valued mapping. $M$ is said to be $H(\cdot, \cdot)$-cocoercive with respect to mappings $A$ and $B$ (or simply $H(\cdot, \cdot)$-cocoercive in the sequel) if $M$ is cocoercive and $(H(A, B)+\lambda M)(X)=X$, for every $\lambda>0$.

Example 6 (see [1]). Let $X, A, B$, and $H$ be the same as in Example 4 , and let $M: \mathbb{R}^{2} \rightarrow \mathbb{R}^{2}$ be defined by $M\left(x_{1}, x_{2}\right)=$ $\left(0, x_{2}\right)$, for all $\left(x_{1}, x_{2}\right) \in \mathbb{R}^{2}$. Then $M$ is cocoercive and $(H(A, B)+\lambda M)\left(\mathbb{R}^{2}\right)=\mathbb{R}^{2}$, for all $\lambda>0$; that is, $M$ is $H(\cdot, \cdot)$ cocoercive with respect to $A$ and $B$.

Proposition 7 (see [1]). Let $H(A, B)$ be $\mu$-cocoercive with respect to $A$ and $\gamma$-relaxed cocoercive with respect to $B$. $A$ is $\alpha$ expansive, $B$ is $\beta$-Lipschitz continuous, and $\mu>\gamma, \alpha>\beta$. Let $M: X \rightarrow 2^{X}$ be $H(\cdot, \cdot)$-cocoercive operator. If the following inequality

$$
\langle x-y, u-v\rangle \geq 0
$$

holds for all $(v, y) \in \operatorname{Graph}(M)$, then $x \in M u$, where

$$
\operatorname{Graph}(M)=\{(x, u) \in X \times X: u \in M(x)\} .
$$

Theorem 8 (see [1]). Let $H(A, B)$ be a $\mu$-cocoercive with respect to $A$ and $\gamma$-relaxed cocoercive with respect to $B$. $A$ is $\alpha$-expansive, $B$ is $\beta$-Lipschitz continuous, and $\mu>\gamma, \alpha>\beta$. Let $M$ be an $H(\cdot, \cdot)$-cocoercive operator with respect to $A$ and $B$. Then the operator $(H(A, B)+\lambda M)^{-1}$ is single-valued.

Definition 9 (see [1]). Let $H(A, B)$ be a $\mu$-cocoercive with respect to $A$ and $\gamma$-relaxed cocoercive with respect to $B$. $A$ is $\alpha$-expansive, $B$ is $\beta$-Lipschitz continuous, and $\mu>\gamma, \alpha>\beta$. Let $M$ be an $H(\cdot, \cdot)$-cocoercive operator with respect to $A$ and $B$. The resolvent operator $R_{\lambda, M}^{H(\cdot,)}: X \rightarrow X$ is defined by

$$
R_{\lambda, M}^{H(\cdot \cdot)}(u)=(H(A, B)+\lambda M)^{-1}(u), \quad \forall u \in X .
$$

Theorem 10 (see [1]). Let $H(A, B)$ be a $\mu$-cocoercive with respect to $A$ and $\gamma$-relaxed cocoercive with respect to $B$. $A$ is $\alpha$-expansive, $B$ is $\beta$-Lipschitz continuous, and $\mu>\gamma, \alpha>\beta$. Let $M$ be an $H(\cdot, \cdot)$-cocoercive operator with respect to $A$ and $B$. Then resolvent operator $R_{\lambda, M}^{H(\cdot,)}: X \rightarrow X$ is $1 /\left(\mu \alpha^{2}-\right.$ $\left.\gamma \beta^{2}\right)$-Lipschitz continuous; that is,

$$
\left\|R_{\lambda, M}^{H(\cdot, \cdot)}(u)-R_{\lambda, M}^{H(\cdot, \cdot)}(v)\right\| \leq \frac{1}{\mu \alpha^{2}-\gamma \beta^{2}}\|u-v\|, \quad \forall u, v \in X .
$$

\section{A New System of Generalized Variational Inclusions}

In this section, we will introduce a new system of generalized variational inclusions involving $H(\cdot, \cdot)$-cocoercive operators.

Let $X$ be a real Hilbert space whose inner product and norm are denoted by $\langle\cdot, \cdot\rangle,\|\cdot\|$, respectively. Let $C$ be a closed and convex set in $X$. Let $H, T_{1}, T_{2}: X \times X \rightarrow X$, and $A, B, g, h: X \rightarrow X$ be single-valued mappings. Let 
$M: X \rightarrow 2^{X}$ be a set-valued mapping such that $M$ is $H(\cdot, \cdot)$ cocoercive operator with respect to $A$ and $B$ and $\varphi: X \rightarrow$ $R \cup\{+\infty\}$ be a continuous function. We consider the system of generalized variational inclusions of finding $\left(x^{*}, y^{*}\right) \in X$ such that

$$
\begin{array}{ll}
0 \in \rho T_{1}\left(y^{*}, x^{*}\right)+\rho M\left(g\left(x^{*}\right)\right)-g\left(y^{*}\right)+g\left(x^{*}\right), & \rho>0, \\
0 \in \eta T_{2}\left(x^{*}, y^{*}\right)+\eta M\left(h\left(x^{*}\right)\right)-h\left(y^{*}\right)+h\left(x^{*}\right), & \eta>0 .
\end{array}
$$

Special Cases. (I) If $T_{1}, T_{2}: X \rightarrow X$ are univariate mappings, problem (16) is equivalent to finding $\left(x^{*}, y^{*}\right) \in X$, such that

$$
\begin{array}{ll}
0 \in \rho T_{1}\left(y^{*}\right)+\rho M\left(g\left(x^{*}\right)\right)-g\left(y^{*}\right)+g\left(x^{*}\right), & \rho>0, \\
0 \in \eta T_{2}\left(x^{*}\right)+\eta M\left(h\left(x^{*}\right)\right)-h\left(y^{*}\right)+h\left(x^{*}\right), & \eta>0,
\end{array}
$$

which appears to be a new one.

(II) If $T_{1}=T_{2}=T$, problem (16) is equivalent to finding $\left(x^{*}, y^{*}\right) \in X$, such that

$$
\begin{array}{ll}
0 \in \rho T\left(y^{*}, x^{*}\right)+\rho M\left(g\left(x^{*}\right)\right)-g\left(y^{*}\right)+g\left(x^{*}\right), & \rho>0, \\
0 \in \eta T\left(x^{*}, y^{*}\right)+\eta M\left(h\left(x^{*}\right)\right)-h\left(y^{*}\right)+h\left(x^{*}\right), & \eta>0,
\end{array}
$$

which appear to be a new one.

(III) If $T_{1}=T_{2}=T, \rho=\eta, g=h$, and $x^{*}=y^{*}=x$, problem (17) is equivalent to finding $x \in X$, such that

$$
0 \in T(x)+M(g(x)),
$$

which is known as the variational inclusion problem or finding the zero of the sum of two (more) cocoercive operators. It is well known that a wide class of linear and nonlinear problems can be studied via variational inclusion problems.

(IV) We note that if $M(\cdot)=\partial \varphi(\cdot)$, the subdifferential of a proper, convex and lower semicontinuous function, then the system of variational inclusions (16) is equivalent to finding $\left(x^{*}, y^{*}\right) \in X$ such that

$$
\begin{array}{ll}
0 \in \rho T_{1}\left(y^{*}, x^{*}\right)+\rho \partial \varphi\left(g\left(x^{*}\right)\right)-g\left(y^{*}\right)+g\left(x^{*}\right), & \rho>0, \\
0 \in \eta T_{2}\left(x^{*}, y^{*}\right)+\eta \partial \varphi\left(h\left(x^{*}\right)\right)-h\left(y^{*}\right)+h\left(x^{*}\right), & \eta>0
\end{array}
$$

or equivalently the problem of finding $\left(x^{*}, y^{*}\right) \in X$ such that

$$
\begin{array}{r}
\left\langle\rho T_{1}\left(y^{*}, x^{*}\right)+g\left(x^{*}\right)-g\left(y^{*}\right), x-g\left(x^{*}\right)\right\rangle \\
\geq \rho \varphi\left(g\left(x^{*}\right)\right)-\rho \varphi(x), \quad x \in X, \rho>0, \\
\left\langle\eta T_{2}\left(y^{*}, x^{*}\right)+h\left(y^{*}\right)-h\left(x^{*}\right), x-h\left(y^{*}\right)\right\rangle \\
\geq \eta \varphi\left(h\left(y^{*}\right)\right)-\eta \varphi(x), \quad x \in X, \eta>0,
\end{array}
$$

which is called the system of mixed general variational inequalities involving four different nonlinear operators. The problem of type (21) is studied in [7].
(V) If $T_{1}=T_{2}=T$ is univariate operator and $g=h, \rho=\eta$, and $x^{*}=y^{*}=x$, problem (21) is equivalent to finding $x \in X$, such that

$$
\langle T x, y-g(x)\rangle \geq \varphi(g(x))-\varphi(y), \quad \forall y \in X,
$$

which is known as the mixed general variational inequality or variational inequality of the second type. For the applications and numerical methods for solving the mixed variational inequalities, see [12].

(VI) If $\varphi$ is an indicator function of a closed convex set $C$ in $X$, then problem (21) is equivalent to finding $\left(x^{*}, y^{*}\right) \in X$ : $g\left(x^{*}\right), h\left(y^{*}\right) \in C$ such that

$$
\begin{aligned}
& \left\langle\rho T_{1}\left(y^{*}, x^{*}\right)+g\left(x^{*}\right)-g\left(y^{*}\right), x-g\left(x^{*}\right)\right\rangle \\
& \geq 0, \quad \forall x \in C, \rho>0, \\
& \left\langle\eta T_{2}\left(x^{*}, y^{*}\right)+h\left(y^{*}\right)-h\left(x^{*}\right), x-h\left(y^{*}\right)\right\rangle \\
& \geq 0, \quad \forall x \in C, \eta>0,
\end{aligned}
$$

which is called the system of general variational inequalities. Such type of problem is studied in [20].

(VII) If $T_{1}=T_{2}=T$, then problem (21) is equivalent to finding $\left(x^{*}, y^{*}\right) \in X: g\left(x^{*}\right), h\left(y^{*}\right) \in C$ such that

$$
\begin{aligned}
& \left\langle\rho T\left(y^{*}, x^{*}\right)+g\left(x^{*}\right)-g\left(y^{*}\right), x-g\left(x^{*}\right)\right\rangle \\
& \quad \geq 0, \quad \forall x \in C, \rho>0, \\
& \left\langle\eta T\left(x^{*}, y^{*}\right)+h\left(y^{*}\right)-h\left(x^{*}\right), x-h\left(y^{*}\right)\right\rangle \\
& \quad \geq 0, \quad \forall x \in C, \eta>0,
\end{aligned}
$$

which can be viewed as a generalization of the system considered and studied in $[17,21]$.

(VIII) If $\varphi(\cdot)$ is the indicator function of a closed convex set $C$, then problem (22) is equivalent to finding $x^{*} \in X$ : $g\left(x^{*}\right) \in C$ such that

$$
\left\langle T x^{*}, x-g\left(x^{*}\right)\right\rangle \geq 0, \quad \forall x \in K,
$$

which is known as the general variational inequality introduced and studied by Noor [22, 23] in 1988. This shows that the system of generalized variational inclusions (16) is more general and includes several classes of variational inclusions/inequalities and related optimization problems as special cases. For the recent applications, numerical methods, and formulations of variational inequalities and variational inclusions, see [1-24] and the references therein.

We now show that the system of generalized variational inclusions (16) is equivalent to the fixed-point problem, and this is the motivation of our next result.

Lemma 11. Let $M$ be $H(\cdot, \cdot)$-cocoercive operator. Then $\left(x^{*}, y^{*}\right) \in X$ is a solution of problem (16) if and only if $\left(x^{*}, y^{*}\right) \in X$ satisfies the following:

$$
\begin{aligned}
& g\left(x^{*}\right)=R_{\rho M}^{H(\cdot, \cdot)}\left[H\left(A\left(g\left(y^{*}\right)\right), B\left(g\left(y^{*}\right)\right)\right)-\rho T_{1}\left(y^{*}, x^{*}\right)\right], \\
& h\left(y^{*}\right)=R_{\eta M}^{H(\cdot,)}\left[H\left(A\left(h\left(x^{*}\right)\right), B\left(h\left(x^{*}\right)\right)\right)-\eta T_{2}\left(x^{*}, y^{*}\right)\right] .
\end{aligned}
$$


where $R_{\rho M}^{H(\cdot, \cdot)}(u)=(H(A, B)+\rho M)^{-1}(u)$ and $R_{\eta M}^{H(\cdot, \cdot)}(u)=$ $(H(A, B)+\eta M)^{-1}(u)$.

Proof. The conclusion can be drawn directly from the definition of resolvent operators $R_{\rho M}^{H(\cdot, \cdot)}$ and $R_{\eta M}^{H(\cdot, \cdot)}$.

This equivalent formulation is used to suggest and analyze a number of iterative methods for solving the system of generalized variational inclusions (16). To do so, one rewrites the equations in the following form:

$$
\begin{aligned}
x^{*}= & x^{*}-g\left(x^{*}\right) \\
& +R_{\rho M}^{H(\cdot, \cdot)}\left[H\left(A\left(g\left(y^{*}\right)\right), B\left(g\left(y^{*}\right)\right)\right)-\rho T_{1}\left(y^{*}, x^{*}\right)\right], \\
y^{*}= & y^{*}-h\left(y^{*}\right) \\
& +R_{\eta M}^{H(\cdot, \cdot)}\left[H\left(A\left(h\left(x^{*}\right)\right), B\left(h\left(x^{*}\right)\right)\right)-\eta T_{2}\left(x^{*}, y^{*}\right)\right] .
\end{aligned}
$$

Based on Lemma 11, we construct the following iterative algorithm for solving (16).

Algorithm 12. For a given $\left(x_{0}, y_{0}\right) \in X$, compute the sequences $\left\{x_{n}\right\}$ and $\left\{y_{n}\right\}$ from the iterative schemes:

$$
\begin{aligned}
x_{n+1}= & \left(1-\omega_{n}\right) x_{n}+\omega_{n}\left(x_{n}-g\left(x_{n}\right)\right) \\
& +\omega_{n} R_{\rho M}^{H(\cdot, \cdot)}\left[H\left(A\left(g\left(y_{n}\right)\right), B\left(g\left(y_{n}\right)\right)\right)\right. \\
& \left.-\rho T_{1}\left(y_{n}, x_{n}\right)\right], \quad n \geq 0, \\
y_{n}= & y_{n}-h\left(y_{n}\right) \\
& +R_{\eta M}^{H(\cdot \cdot)}\left[H\left(A\left(h\left(x_{n}\right)\right), B\left(h\left(x_{n}\right)\right)\right)\right. \\
& \left.\quad-\eta T_{2}\left(x_{n}, y_{n}\right)\right], \quad n \geq 1,
\end{aligned}
$$

where $\omega_{n} \in[0,1]$.

If $\omega_{n}=1$, then Algorithm 12 reduces to Algorithm 13 .

Algorithm 13. For a given $\left(x_{0}, y_{0}\right) \in X$, compute the sequences $\left\{x_{n}\right\}$ and $\left\{y_{n}\right\}$ from the iterative schemes:

$$
\begin{aligned}
x_{n+1}= & x_{n}-g\left(x_{n}\right) \\
& +R_{\rho M}^{H(\cdot, \cdot)}\left[H\left(A\left(g\left(y_{n}\right)\right), B\left(g\left(y_{n}\right)\right)\right)\right. \\
& \left.\quad-\rho T_{1}\left(y_{n}, x_{n}\right)\right], \quad n \geq 0, \\
h\left(y_{n}\right)= & \quad R_{\eta M}^{H(\cdot, \cdot)}\left[H\left(A\left(h\left(x_{n}\right)\right), B\left(h\left(x_{n}\right)\right)\right)\right. \\
& \left.-\eta T_{2}\left(x_{n}, y_{n}\right)\right], \quad n \geq 1 .
\end{aligned}
$$

For suitable and appropriate choice of the operators $M$, $H, T_{1}, T_{2}, A, B, g, h$, and spaces, one can obtain a wide class of iterative methods for solving different classes of variational inclusions and related optimization problems. This shows that Algorithm 12 is quite flexible and general and includes various known and new algorithms for solving variational inequalities and related optimization problems as special cases.

Definition 14. A mapping $T: X \times X \rightarrow X$ is called $p$-strongly monotone in the first variable if there exists a constant $p>0$ such that, for all $x, y \in X$,

$$
\left\langle T\left(x, x^{\prime}\right)-T\left(y, y^{\prime}\right), x-y\right\rangle \geq p\|x-y\|^{2}, \quad \forall x^{\prime}, y^{\prime} \in X .
$$

Definition 15. A mapping $T: X \times X \rightarrow X$ is called relaxed $q$-cocoercive if there exists a constant $q>0$ such that, for all $x, y \in X$,

$$
\begin{aligned}
& \left\langle T\left(x, x^{\prime}\right)-T\left(y, y^{\prime}\right), x-y\right\rangle \\
& \quad \geq-q\left\|T\left(x, x^{\prime}\right)-T\left(y, y^{\prime}\right)\right\|^{2}, \quad \forall x^{\prime}, y^{\prime} \in X .
\end{aligned}
$$

Definition 16. A mapping $\mathrm{T}: X \times X \rightarrow X$ is called relaxed $(p, q)$-cocoercive in the first variable if there exist constants $p>0, q>0$ such that, for all $x, y \in X$,

$$
\begin{aligned}
& \left\langle T\left(x, x^{\prime}\right)-T\left(y, y^{\prime}\right), x-y\right\rangle \\
& \quad \geq-p\left\|T\left(x, x^{\prime}\right)-T\left(y, y^{\prime}\right)\right\|^{2}+q\|x-y\|^{2}, \quad \forall x^{\prime}, y^{\prime} \in X .
\end{aligned}
$$

The class of relaxed $(p, q)$-cocoercive mappings is more general than the class of strongly monotone mappings.

Definition 17. A mapping $T: X \times X \rightarrow X$ is called $r$-Lipschitz continuous in the first variable if there exists a constant $r>0$ such that, for all $x, y \in X$,

$$
\left\|T\left(x, x^{\prime}\right)-T\left(y, y^{\prime}\right)\right\| \leq r\|x-y\|, \quad \forall x^{\prime}, y^{\prime} \in X .
$$

Lemma 18 (see [24]). Assume that $\left\{a_{n}\right\}$ is a sequence of nonnegative real numbers such that

$$
a_{n+1} \leq\left(1-\lambda_{n}\right) a_{n}+b_{n}, \quad \forall n \geq 0,
$$

where $\lambda_{n}$ is a sequence in $[0,1]$ with $\sum_{n=0}^{\infty} \lambda_{n}=\infty, b_{n}=o\left(\lambda_{n}\right)$, and then $\lim _{n \rightarrow \infty} a_{n}=0$.

Theorem 19. Let $X$ be a real Hilbert space. Suppose that $H, T_{1}, T_{2}: X \times X \rightarrow X, A, B, g, h: X \rightarrow X$ are singlevalued mappings and $M: X \rightarrow 2^{X}$ is a set-valued mapping such that $M$ is $H(\cdot, \cdot)$-cocoercive operator with respect to $A$ and $B$. Assume that

(i) $H(A, B)$ is $\mu$-cocoercive with respect to $A, \gamma$-relaxed cocoercive with respect to $B$, and $\mu>\gamma$;

(ii) $A$ is $\alpha$-expansive, $B$ is $\beta$-Lipschitz continuous, and $\alpha>$ $\beta$;

(iii) $H(A, B)$ is $\delta_{1}$-Lipschitz continuous with respect to $A$ and $\delta_{2}$-Lipschitz continuous with respect to $B$;

(iv) $T_{1}: X \times X \rightarrow X$ is relaxed $\left(p_{1}, q_{1}\right)$-cocoercive and $r_{1}$-Lipschitz continuous in the first variable; 
(v) $T_{2}: X \times X \rightarrow X$ is relaxed $\left(p_{2}, q_{2}\right)$-cocoercive and $r_{2}$-Lipschitz continuous in the first variable;

(vi) $g: X \rightarrow X$ is relaxed $\left(s_{1}, t_{1}\right)$-cocoercive and $l_{1}$ Lipschitz continuous;

(vii) $h: X \rightarrow X$ is relaxed $\left(s_{2}, t_{2}\right)$-cocoercive and $l_{2}$ Lipschitz continuous;

(viii) $\omega_{n} \in[0,1]$ and $\sum_{n=0}^{\infty} \omega_{n}=\infty$;

(ix) $\theta_{4}<1$ and $\left(1-\theta_{3}\right)\left(1-\theta_{4}\right)>L_{n}^{2}\left(\theta_{1}+\theta_{5}\right)\left(\theta_{2}+\theta_{6}\right)$, where

$$
\begin{gathered}
\theta_{1}=\sqrt{1+2 \rho p_{1} r_{1}^{2}-2 \rho q_{1}+\rho^{2} r_{1}^{2}}, \\
\theta_{2}=\sqrt{1+2 \eta p_{2} r_{2}^{2}-2 \eta q_{2}+\eta^{2} r_{2}^{2}}, \\
\theta_{3}=\sqrt{1+2 s_{1} l_{1}^{2}-2 t_{1}+l_{1}^{2}}, \\
\theta_{4}=\sqrt{1+2 s_{2} l_{2}^{2}-2 t_{2}+l_{2}^{2}}, \\
\theta_{5}=\sqrt{1+l_{1}^{2} \delta_{1}^{2}+l_{1}^{2} \delta_{2}^{2}-2 l_{1}^{2} r}, \\
\theta_{6}=\sqrt{1+l_{2}^{2} \delta_{1}^{2}+l_{2}^{2} \delta_{2}^{2}-2 l_{2}^{2} r}, \\
\text { and } r=\mu \alpha^{2}-\gamma \beta^{2}, L_{n}=\left(1 /\left(\mu \alpha^{2}-\gamma \beta^{2}\right)\right) .
\end{gathered}
$$

Then the iterative sequences $\left\{x_{n}\right\}$ and $\left\{y_{n}\right\}$ generated by Algorithm 12 converge strongly to $x^{*}$ and $y^{*}$, respectively, and $\left(x^{*}, y^{*}\right)$ is a solution of problem (16).

Proof. To prove the result, we need first to evaluate $\left\|x_{n+1}-x^{*}\right\|$ for all $n \geq 0$. From (28), and the Lipschitz continuity of the resolvent operator $R_{\rho M}^{H(\cdot,)}$, we have

$$
\begin{aligned}
\| x_{n+1}- & x^{*} \| \\
=\| & \left(1-\omega_{n}\right) x_{n}+\omega_{n} \\
\times & {\left[x_{n}-g\left(x_{n}\right)\right.} \\
& \left.+R_{\rho M}^{H(\cdot, \cdot)}\left[H\left(A\left(g\left(y_{n}\right)\right), B\left(g\left(y_{n}\right)\right)\right)-\rho T_{1}\left(y_{n}, x_{n}\right)\right]\right]-x^{*} \| \\
=\| & \left(1-\omega_{n}\right) x_{n}+\omega_{n} \\
& \times\left[x_{n}-g\left(x_{n}\right)\right. \\
& \left.+R_{\rho M}^{H(\cdot, \cdot)}\left[H\left(A\left(g\left(y_{n}\right)\right), B\left(g\left(y_{n}\right)\right)\right)-\rho T_{1}\left(y_{n}, x_{n}\right)\right]\right] \\
- & \left(1-\omega_{n}\right) x^{*}-\omega_{n} \\
\times & {\left[x^{*}-g\left(x^{*}\right)\right.} \\
+ & \left.R_{\rho M}^{H(\cdot, \cdot)}\left[H\left(A\left(g\left(y^{*}\right)\right), B\left(g\left(y^{*}\right)\right)\right)-\rho T_{1}\left(y^{*}, x^{*}\right)\right]\right] \| \\
\leq & \left(1-\omega_{n}\right)\left\|x_{n}-x^{*}\right\| \\
+ & \omega_{n}\left\|x_{n}-x^{*}-\left(g\left(x_{n}\right)-g\left(x^{*}\right)\right)\right\| \\
+ & \frac{\omega_{n}}{\mu \alpha^{2}-\gamma \beta^{2}} \\
\times & {\left[\| y_{n}-y^{*}\right.} \\
& \quad-\left[H\left(A\left(g\left(y_{n}\right)\right), B\left(g\left(y_{n}\right)\right)\right)\right. \\
& \left.+\left\|y_{n}-y^{*}-\rho\left[T_{1}\left(y_{n}, x_{n}\right)-T_{1}\left(y^{*}, x^{*}\right)\right]\right\|\right] .
\end{aligned}
$$

By the assumption that $T_{1}$ is relaxed $\left(p_{1}, q_{1}\right)$-cocoercive and $r_{1}$-Lipschitz continuous in the first variable, we obtain that

$$
\begin{aligned}
\| y_{n}- & y^{*}-\rho\left[T_{1}\left(y_{n}, x_{n}\right)-T_{1}\left(y^{*}, x^{*}\right)\right] \|^{2} \\
= & \left\|y_{n}-y^{*}\right\|^{2}-2 \rho\left\langle T_{1}\left(y_{n}, x_{n}\right)-T_{1}\left(y^{*}, x^{*}\right), y_{n}-y^{*}\right\rangle \\
& +\rho^{2}\left\|T_{1}\left(y_{n}, x_{n}\right)-T_{1}\left(y^{*}, x^{*}\right)\right\|^{2} \\
\leq & \left\|y_{n}-y^{*}\right\|^{2} \\
& -2 \rho\left[-p_{1}\left\|T_{1}\left(y_{n}, x_{n}\right)-T_{1}\left(y^{*}, x^{*}\right)\right\|^{2}+q_{1}\left\|y_{n}-y^{*}\right\|^{2}\right] \\
& +\rho^{2}\left\|T_{1}\left(y_{n}, x_{n}\right)-T_{1}\left(y^{*}, x^{*}\right)\right\|^{2} \\
\leq & \left\|y_{n}-y^{*}\right\|^{2}+2 \rho p_{1} r_{1}^{2}\left\|y_{n}-y^{*}\right\|^{2} \\
& \quad-2 \rho q_{1}\left\|y_{n}-y^{*}\right\|^{2}+\rho^{2} r_{1}^{2}\left\|y_{n}-y^{*}\right\|^{2} \\
= & \theta_{1}^{2}\left\|y_{n}-y^{*}\right\|^{2},
\end{aligned}
$$

where $\theta_{1}=\sqrt{1+2 \rho p_{1} r_{1}^{2}-2 \rho q_{1}+\rho^{2} r_{1}^{2}}$. By the assumption that $g$ is relaxed $\left(s_{1}, t_{1}\right)$-cocoercive and $l_{1}$-Lipschitz continuous, we arrive at

$$
\begin{aligned}
\| x_{n}- & x^{*}-\left(g\left(x_{n}\right)-g\left(x^{*}\right)\right) \|^{2} \\
= & \left\|x_{n}-x^{*}\right\|^{2}-2\left\langle g\left(x_{n}\right)-g\left(x^{*}\right), x_{n}-x^{*}\right\rangle \\
& +\left\|g\left(x_{n}\right)-g\left(x^{*}\right)\right\|^{2} \\
\leq & \left\|x_{n}-x^{*}\right\|^{2} \\
& -2\left[-s_{1}\left\|g\left(x_{n}\right)-g\left(x^{*}\right)\right\|^{2}+t_{1}\left\|x_{n}-x^{*}\right\|^{2}\right] \\
& +\left\|g\left(x_{n}\right)-g\left(x^{*}\right)\right\|^{2} \\
\leq & \left\|x_{n}-x^{*}\right\|^{2}+2 s_{1} l_{1}^{2}\left\|x_{n}-x^{*}\right\|^{2} \\
& -2 t_{1}\left\|x_{n}-x^{*}\right\|^{2}+l_{1}^{2}\left\|x_{n}-x^{*}\right\|^{2} \\
= & \theta_{3}^{2}\left\|x_{n}-x^{*}\right\|^{2},
\end{aligned}
$$

where $\theta_{3}=\sqrt{1+2 s_{1} l_{1}^{2}-2 t_{1}+l_{1}^{2}}$. Now, we estimate

$$
\begin{aligned}
& \| y_{n}-y^{*} \\
& -\left[H\left(A\left(g\left(y_{n}\right)\right), B\left(g\left(y_{n}\right)\right)\right)\right. \\
& \left.-H\left(A\left(g\left(y^{*}\right)\right), B\left(g\left(y^{*}\right)\right)\right)\right] \|^{2} \\
& \leq\left\|y_{n}-y^{*}\right\|^{2} \\
& -2\left\langle H\left(A\left(g\left(y_{n}\right)\right), B\left(g\left(y_{n}\right)\right)\right)\right. \\
& \left.\quad-H\left(A\left(g\left(y^{*}\right)\right), B\left(g\left(y^{*}\right)\right)\right), y_{n}-y^{*}\right\rangle \\
& +\| H\left(A\left(g\left(y_{n}\right)\right), B\left(g\left(y_{n}\right)\right)\right) \\
& -H\left(A\left(g\left(y^{*}\right)\right), B\left(g\left(y^{*}\right)\right)\right) \|^{2} \\
& \leq\left\|y_{n}-y^{*}\right\|^{2} \\
& -2\left\langle H\left(A\left(g\left(y_{n}\right)\right), B\left(g\left(y_{n}\right)\right)\right)\right. \\
& \left.-H\left(A\left(g\left(y_{n}\right)\right), B\left(g\left(y^{*}\right)\right)\right), y_{n}-y^{*}\right\rangle \\
& -2\left\langle H\left(A\left(g\left(y_{n}\right)\right), B\left(g\left(y^{*}\right)\right)\right)\right.
\end{aligned}
$$




$$
\begin{aligned}
& \left.-H\left(A\left(g\left(y^{*}\right)\right), B\left(g\left(y^{*}\right)\right)\right), y_{n}-y^{*}\right\rangle \\
+ & \| H\left(A\left(g\left(y_{n}\right)\right), B\left(g\left(y_{n}\right)\right)\right) \\
& -H\left(A\left(g\left(y_{n}\right)\right), B\left(g\left(y^{*}\right)\right)\right) \|^{2} \\
+ & \| H\left(A\left(g\left(y^{*}\right)\right), B\left(g\left(y_{n}\right)\right)\right) \\
& -H\left(A\left(g\left(y^{*}\right)\right), B\left(g\left(y^{*}\right)\right)\right) \|^{2} \\
\leq & \left\|y_{n}-y^{*}\right\|^{2}-2 \mu \alpha^{2}\left\|g\left(y_{n}\right)-g\left(y^{*}\right)\right\|^{2} \\
& +2 \gamma \beta^{2}\left\|g\left(y_{n}\right)-g\left(y^{*}\right)\right\|^{2} \\
& +\delta_{1}^{2}\left\|g\left(y_{n}\right)-g\left(y^{*}\right)\right\|^{2}+\delta_{2}^{2}\left\|g\left(y_{n}\right)-g\left(y^{*}\right)\right\|^{2} \\
\leq & {\left[1+l_{1}^{2} \delta_{1}^{2}+l_{1}^{2} \delta_{2}^{2}-2 l_{1}^{2}\left(\mu \alpha^{2}-\gamma \beta^{2}\right)\right]\left\|y_{n}-y^{*}\right\|^{2} } \\
= & {\left[1+l_{1}^{2} \delta_{1}^{2}+l_{1}^{2} \delta_{2}^{2}-2 l_{1}^{2} r\right]\left\|y_{n}-y^{*}\right\|^{2} } \\
= & \theta_{5}^{2}\left\|y_{n}-y^{*}\right\|^{2},
\end{aligned}
$$

where $\theta_{5}=\sqrt{1+l_{1}^{2} \delta_{1}^{2}+l_{1}^{2} \delta_{2}^{2}-2 l_{1}^{2} r}$ and $r=\mu \alpha^{2}-\gamma \beta^{2}$.

Substituting (37)-(39) into (36) yields

$$
\begin{aligned}
\left\|x_{n+1}-x^{*}\right\| \leq & {\left[1-\omega_{n}\left(1-\theta_{3}\right)\right]\left\|x_{n}-x^{*}\right\| } \\
& +\omega_{n} L_{n}\left(\theta_{1}+\theta_{5}\right)\left\|y_{n}-y^{*}\right\|,
\end{aligned}
$$

where $L_{n}=\left(1 /\left(\mu \alpha^{2}-\gamma \beta^{2}\right)\right)$.

Next we estimate

$$
\begin{aligned}
& \left\|y_{n}-y^{*}\right\| \\
& =\| y_{n}-h\left(y_{n}\right) \\
& \quad+R_{\eta M}^{H(\cdot \cdot \cdot)}\left[H\left(A\left(h\left(x_{n}\right)\right), B\left(h\left(x_{n}\right)\right)\right)-\eta T_{2}\left(x_{n}, y_{n}\right)\right] \\
& \quad-y^{*}+h\left(y^{*}\right) \\
& \quad-R_{\eta M}^{H(\cdot, \cdot)}\left[H\left(A\left(h\left(x^{*}\right)\right), B\left(h\left(x^{*}\right)\right)\right)-\eta T_{2}\left(x^{*}, y^{*}\right)\right] \| \\
& \leq\left\|y_{n}-y^{*}-\left(h\left(y_{n}\right)-h\left(y^{*}\right)\right)\right\| \\
& +\frac{1}{\mu \alpha^{2}-\gamma \beta^{2}} \\
& \times\left[\| x_{n}-x^{*}\right. \\
& \quad-\left[H\left(A\left(h\left(x_{n}\right)\right), B\left(h\left(x_{n}\right)\right)\right)\right. \\
& \left.\quad-H\left(A\left(h\left(x^{*}\right)\right), B\left(h\left(x^{*}\right)\right)\right)\right] \| \\
& \left.\quad+\left\|x_{n}-x^{*}-\eta\left[T_{2}\left(x_{n}, y_{n}\right)-T_{2}\left(x^{*}, y^{*}\right)\right]\right\|\right] .
\end{aligned}
$$

By the assumption that $T_{2}$ is relaxed $\left(p_{2}, q_{2}\right)$-cocoercive and $r_{2}$-Lipschitz continuous in the first variable, we see that

$$
\begin{aligned}
\| x_{n}- & x^{*}-\eta\left[T_{2}\left(x_{n}, y_{n}\right)-T_{2}\left(x^{*}, y^{*}\right)\right] \|^{2} \\
= & \left\|x_{n}-x^{*}\right\|^{2}-2 \eta\left\langle T_{2}\left(x_{n}, y_{n}\right)-T_{2}\left(x^{*}, y^{*}\right), x_{n}-x^{*}\right\rangle \\
& +\eta^{2}\left\|T_{2}\left(x_{n}, y_{n}\right)-T_{2}\left(x^{*}, y^{*}\right)\right\|^{2} \\
\leq & \left\|x_{n}-x^{*}\right\|^{2} \\
& -2 \eta\left[-p_{2}\left\|T_{2}\left(x_{n}, y_{n}\right)-T_{2}\left(x^{*}, y^{*}\right)\right\|^{2}+q_{2}\left\|x_{n}-x^{*}\right\|^{2}\right] \\
& +\eta^{2}\left\|T_{2}\left(x_{n}, y_{n}\right)-T_{2}\left(x^{*}, y^{*}\right)\right\|^{2}
\end{aligned}
$$

$$
\begin{aligned}
\leq & \left\|x_{n}-x^{*}\right\|^{2}+2 \eta p_{2} r_{2}^{2}\left\|x_{n}-x^{*}\right\|^{2} \\
& -2 \eta q_{2}\left\|x_{n}-x^{*}\right\|^{2}+\eta^{2} r_{2}^{2}\left\|x_{n}-x^{*}\right\|^{2} \\
= & \theta_{2}^{2}\left\|x_{n}-x^{*}\right\|^{2},
\end{aligned}
$$

where $\theta_{2}=\sqrt{1+2 \eta p_{2} r_{2}^{2}-2 \eta q_{2}+\eta^{2} r_{2}^{2}}$. From the proof of (38), we can obtain that

$$
\left\|y_{n}-y^{*}-\left(h\left(y_{n}\right)-h\left(y^{*}\right)\right)\right\|^{2} \leq \theta_{4}^{2}\left\|y_{n}-y^{*}\right\|^{2},
$$

where $\theta_{4}=\sqrt{1+2 s_{2} l_{2}^{2}-2 t_{2}+l_{2}^{2}}$.

Now, we estimate

$$
\begin{aligned}
\| x_{n}-x^{*} & \\
-[H & \left.H\left(h\left(x_{n}\right)\right), B\left(h\left(x_{n}\right)\right)\right) \\
- & \left.H\left(A\left(h\left(x^{*}\right)\right), B\left(h\left(x^{*}\right)\right)\right)\right] \|^{2} \\
\leq & \left\|x_{n}-x^{*}\right\|^{2} \\
& -2\left\langle H\left(A\left(h\left(x_{n}\right)\right), B\left(h\left(x_{n}\right)\right)\right)\right. \\
& \left.-H\left(A\left(h\left(x^{*}\right)\right), B\left(h\left(x^{*}\right)\right)\right), x_{n}-x^{*}\right\rangle \\
& +\| H\left(A\left(h\left(x_{n}\right)\right), B\left(h\left(x_{n}\right)\right)\right) \\
& -H\left(A\left(h\left(x^{*}\right)\right), B\left(h\left(x^{*}\right)\right)\right) \|^{2} \\
\leq & \left\|x_{n}-x^{*}\right\|^{2} \\
& -2\left\langle H\left(A\left(h\left(x_{n}\right)\right), B\left(h\left(x_{n}\right)\right)\right)\right. \\
& \left.-H\left(A\left(h\left(x^{*}\right)\right), B\left(h\left(x_{n}\right)\right)\right), x_{n}-x^{*}\right\rangle \\
& -2\left\langle H\left(A\left(h\left(x^{*}\right)\right), B\left(h\left(x_{n}\right)\right)\right)\right. \\
& \left.\quad-H\left(A\left(h\left(x^{*}\right)\right), B\left(h\left(x^{*}\right)\right)\right), x_{n}-x^{*}\right\rangle \\
& +\| H\left(A\left(h\left(x^{*}\right)\right), B\left(h\left(x_{n}\right)\right)\right) \\
& -H\left(A\left(h\left(x^{*}\right)\right), B\left(h\left(x_{n}\right)\right)\right) \|^{2} \\
& +\| H\left(A\left(h\left(x^{*}\right)\right), B\left(h\left(x_{n}\right)\right)\right) \\
& -H\left(A\left(h\left(x^{*}\right)\right), B\left(h\left(x^{*}\right)\right)\right) \|^{2} \\
\leq & \left\|x_{n}-x^{*}\right\|^{2}-2 \mu \alpha^{2}\left\|h\left(x_{n}\right)-h\left(x^{*}\right)\right\|^{2} \\
+ & 2 \gamma \beta^{2}\left\|h\left(x_{n}\right)-h\left(x^{*}\right)\right\|^{2} \\
+ & \delta_{1}^{2}\left\|h\left(x_{n}\right)-h\left(x^{*}\right)\right\|^{2}+\delta_{2}^{2}\left\|h\left(x_{n}\right)-h\left(x^{*}\right)\right\|^{2} \\
\leq & \left\|x_{n}-x^{*}\right\|^{2} \\
+ & {\left[\left(\delta_{1}^{2}+\delta_{2}^{2}\right)-2\left(\mu \alpha^{2}-\gamma \beta^{2}\right)\right]\left\|h\left(x_{n}\right)-h\left(x^{*}\right)\right\|^{2} } \\
= & {\left[1+l_{2}^{2} \delta_{1}^{2}+l_{2}^{2} \delta_{2}^{2}-2 l_{2}^{2} r\right]\left\|x_{n}-x^{*}\right\|^{2} } \\
\theta_{6}^{2} & \left\|x_{n}-x^{*}\right\|^{2}, \\
&
\end{aligned}
$$

where $\theta_{6}=\sqrt{1+l_{2}^{2} \delta_{1}^{2}+l_{2}^{2} \delta_{2}^{2}-2 l_{2}^{2} r}$ and $r=\mu \alpha^{2}-\gamma \beta^{2}$. Substituting (42)-(44) into (41) yields

$$
\left\|y_{n}-y^{*}\right\| \leq \theta_{4}\left\|y_{n}-y^{*}\right\|+L_{n}\left(\theta_{2}+\theta_{6}\right)\left\|x_{n}-x^{*}\right\|,
$$


where $L_{n}=\left(1 /\left(\mu \alpha^{2}-\gamma \beta^{2}\right)\right)$. Since $\theta_{4}<1$, we observe that

$$
\left\|y_{n}-y^{*}\right\| \leq \frac{L_{n}\left(\theta_{2}+\theta_{6}\right)}{1-\theta_{4}}\left\|x_{n}-x^{*}\right\| .
$$

Substituting (46) into (40) yields

$$
\begin{aligned}
\left\|x_{n+1}-x^{*}\right\| \leq & {\left[1-\omega_{n}\left(1-\theta_{3}\right)\right]\left\|x_{n}-x^{*}\right\| } \\
& +\omega_{n} L_{n}^{2} \frac{\left(\theta_{1}+\theta_{5}\right)\left(\theta_{2}+\theta_{6}\right)}{1-\theta_{4}}\left\|x_{n}-x^{*}\right\| \\
= & {\left[1-\omega_{n}\left\{1-\theta_{3}-L_{n}^{2} \frac{\left(\theta_{1}+\theta_{5}\right)\left(\theta_{2}+\theta_{6}\right)}{1-\theta_{4}}\right\}\right] } \\
& \times\left\|x_{n}-x^{*}\right\| .
\end{aligned}
$$

Noticing condition (ix) and applying Lemma 18 to (47), we get the desired conclusion easily. This completes the proof.

Example 20. Let $X=\mathbb{R}^{2}$ with usual inner product. Let $A, B$ : $\mathbb{R}^{2} \rightarrow \mathbb{R}^{2}$ be defined by

$$
A x=\left(\begin{array}{l}
7 x_{1} \\
7 x_{2}
\end{array}\right), \quad B x=\left(\begin{array}{c}
-\frac{1}{7} x_{1} \\
-\frac{1}{7} x_{2}
\end{array}\right), \quad \forall x \in \mathbb{R}^{2} .
$$

Suppose that $H: \mathbb{R}^{2} \times \mathbb{R}^{2} \rightarrow \mathbb{R}^{2}$ is defined by

$$
H(A x, B y)=A x+B y, \quad \forall x \in \mathbb{R}^{2} .
$$

Then, it is easy to cheek the following.

(i) $H(A, B)$ is $1 / n$-cocoercive with respect to $A$, for $n=7$, 8 , and $7 / n$-relaxed cocoercive with respect to $B$, for $n=1,2$.

(ii) $A$ is $n$-expansive, for $n=6,7$, and $B$ is $1 / n$-Lipschitz continuous, for $n=6,7$.

(iii) $H(A, B)$ is $n$-Lipschitz continuous with respect to $A$, for $n=7,8$, and $1 / n$-Lipschitz continuous with respect to $B$, for $n=6,7$.

Let $T_{1}, T_{2}: \mathbb{R}^{2} \times \mathbb{R}^{2} \rightarrow \mathbb{R}^{2}$ be defined by

$$
\begin{aligned}
T_{1}\left(x, x^{\prime}\right)=\left(\begin{array}{l}
x_{1} \\
x_{2}
\end{array}\right), \quad T_{2}\left(y, y^{\prime}\right)= & \left(\begin{array}{l}
\frac{1}{2} y_{1}-\frac{1}{4} y_{2}^{\prime} \\
\frac{1}{4} y_{1}+\frac{1}{2} y_{2}^{\prime}
\end{array}\right), \\
& \forall x, x^{\prime}, y, y^{\prime} \in \mathbb{R}^{2} .
\end{aligned}
$$

Then, it is easy to verify the following.

(iv) $T_{1}$ is relaxed $(1,2)$-cocoercive and 1-Lipschitz continuous.

(v) $T_{2}$ is relaxed $(1,(13 / n))$-cocoercive, for $n=16,17$, and $\sqrt{5} / n$-Lipschitz continuous, for $n=3,4$.
Let $g, h: \mathbb{R}^{2} \rightarrow \mathbb{R}^{2}$ be defined by

$$
g(x)=\left(\begin{array}{l}
\frac{1}{2} x_{1} \\
\frac{1}{2} x_{2}
\end{array}\right), \quad h(x)=\left(\begin{array}{l}
\frac{2}{3} x_{1} \\
\frac{2}{3} x_{2}
\end{array}\right), \quad \forall x \in \mathbb{R}^{2} .
$$

Then, it is easy to verify the following.

(vi) $g$ is relaxed $(1,(3 / n))$-cocoercive, for $n=4,5$, and $1 / n$-Lipschitz continuous, for $n=1,2$.

(vii) $h$ is relaxed $(1,(10 / n))$-cocoercive, for $n=9,10$, and $2 / n$-Lipschitz continuous, for $n=2,3$.

(viii) Clearly, for the constants

$$
\begin{gathered}
\mu=\frac{1}{7}, \quad \alpha=7, \quad \gamma=7, \quad \beta=\frac{1}{7}, \quad \delta_{1}=7, \\
\delta_{2}=\frac{1}{7}, \quad L_{n}=\frac{7}{48}, \quad p_{1}=1, \quad q_{1}=2, \\
r_{1}=1, \quad p_{2}=1, \quad q_{2}=\frac{13}{16}, \quad r_{2}=\frac{\sqrt{5}}{4}, \\
s_{1}=1, \quad t_{1}=\frac{3}{4}, \quad l_{1}=\frac{1}{2}, \quad s_{2}=1, \\
t_{2}=\frac{10}{9}, \quad l_{2}=\frac{2}{3}, \quad \theta_{1}=0.1, \quad \theta_{2}=0.56, \\
\theta_{3}=0.5, \quad \theta_{4}=0.33, \quad \theta_{5}=3.13, \quad \theta_{6}=4.08,
\end{gathered}
$$

obtained in (i) to (vii) above, the conditions of Theorem 19 are satisfied for the inclusion system (16) for $\rho=0.9, \eta=1$.

\section{References}

[1] R. Ahmad, M. Dilshad, M.-M. Wong, and J. C. Yao, " $H(\cdot, \cdot)$ cocoercive operator and an applications for solving generalized variational inclusions," Abstract and Applied Analysis, vol. 2011, Article ID 261534, 12 pages, 2011.

[2] C. Bai and Q. Yang, "A system of nonlinear set-valued implicit variational inclusions in real banach spaces," Communications of the Korean Mathematical Society, vol. 25, no. 1, pp. 129-137, 2010.

[3] Y. P. Fang and N. J. Huang, " $H$-monotone operator and resolvent operator technique for variational inclusions," Applied Mathematics and Computation, vol. 145, no. 2-3, pp. 795-803, 2003.

[4] Y. P. Fang and N. J. Huang, " $H$-accretive operators and resolvent operator technique for solving variational inclusions in Banach spaces," Applied Mathematics Letters, vol. 17, no. 6, pp. 647-653, 2004.

[5] Y. P. Fang, Y. J. Cho, and J. K. Kim, “( $H, \eta)$-accretive operator and approximating solutions for systems of variational inclusions in Banach spaces," Applied Mathematics Letters. In press.

[6] Y. P. Fang and N. J. Huang, "Approximate solutions for nonlinear variational inclusions with $(H, \eta)$-monotone operator," Research Report, Sichuan University, Sichuan, China, 2003. 
[7] S. Husain and S. Gupta, "A resolvent operator technique for solving generalized system of nonlinear relaxed cocoercive mixed variational inequalities," Advances in Fixed Point Theory, vol. 2, no. 1, pp. 18-28, 2012.

[8] K. R. Kazmi, M. I. Bhat, and N. Ahmad, "An iterative algorithm based on $M$-proximal mappings for a system of generalized implicit variational inclusions in Banach spaces," Journal of Computational and Applied Mathematics, vol. 233, no. 2, pp. 361-371, 2009.

[9] K. R. Kazmi, N. Ahmad, and M. Shahzad, "Convergence and stability of an iterative algorithm for a system of generalized implicit variational-like inclusions in Banach spaces," Applied Mathematics and Computation, vol. 218, pp. 9208-9219, 2012.

[10] H. Y. Lan, Y. J. Cho, and R. U. Verma, "Nonlinear relaxed cocoercive variational inclusions involving $(A, \eta)$-accretive mappings in banach spaces," Computers and Mathematics with Applications, vol. 51, no. 9-10, pp. 1529-1538, 2006.

[11] M. A. Noor, K. I. Noor, and E. Al-Said, "On a system of general variational inclusions," Advanced Modeling and Optimization, vol. 13, no. 2, pp. 221-231, 2011.

[12] M. A. Noor and K. I. Noor, "Resolvent methods for solving general variational inclusions," Journal of Optimization Theory and Applications, vol. 148, no. 2, pp. 422-430, 2011.

[13] R. U. Verma, "A-monotonicity and its role in nonlinear variational inclusions," Journal of Optimization Theory and Applications, vol. 129, no. 3, pp. 457-467, 2006.

[14] Z. Xu and Z. Wang, "A generalized mixed variational inclusion involving $(H(\cdot, \cdot), \eta)$-monotone operators in Banach spaces," Journal of Mathematics Research, vol. 2, no. 3, pp. 47-56, 2010.

[15] Y. Z. Zou and N. J. Huang, " $H(\cdot, \cdot)$-accretive operator with an application for solving variational inclusions in Banach spaces," Applied Mathematics and Computation, vol. 204, no. 2, pp. 809816, 2008.

[16] J. P. Aubin and A. Cellina, Differential Inclusions, Springer, Berlin, Germany, 1984.

[17] Z. Huang and M. A. Noor, "An explicit projection method for a system of nonlinear variational inequalities with different $(\gamma, r)$ cocoercive mappings," Applied Mathematics and Computation, vol. 190, no. 1, pp. 356-361, 2007.

[18] S. Karamardian, "The nonlinear complementarity problem with applications, part 2," Journal of Optimization Theory and Applications, vol. 4, no. 3, pp. 167-181, 1969.

[19] P. Tseng, "Further applications of a splitting algorithm to decomposition in variational inequalities and convex programming," Mathematical Programming, vol. 48, no. 1-3, pp. 249$263,1990$.

[20] M. A. Noor and K. I. Noor, "Projection algorithms for solving a system of general variational inequalities," Nonlinear Analysis: Theory, Methods and Applications, vol. 70, no. 7, pp. 2700-2706, 2009.

[21] S. S. Chang, H. W. J. Lee, and C. K. Chan, "Generalized system for relaxed cocoercive variational inequalities in Hilbert spaces," Applied Mathematics Letters, vol. 20, no. 3, pp. 329-334, 2006.

[22] M. A. Noor, "Differentiable non-convex functions and general variational inequalities," Applied Mathematics and Computation, vol. 199, no. 2, pp. 623-630, 2008.

[23] M. A. Noor, "Quasi variational inequalities," Applied Mathematics Letters, vol. 1, no. 4, pp. 367-370, 1988.

[24] X. L. Wang, "Fixed point iteration for local strictly pseudocontractive mapping," Proceeding of American Mathematical Society, vol. 113, pp. 727-731, 1991. 


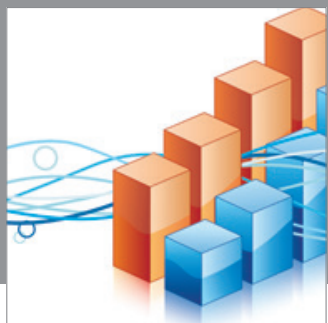

Advances in

Operations Research

mansans

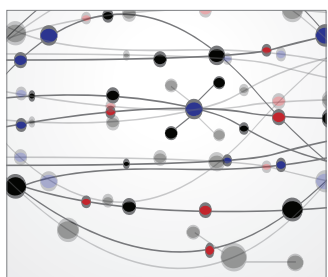

The Scientific World Journal
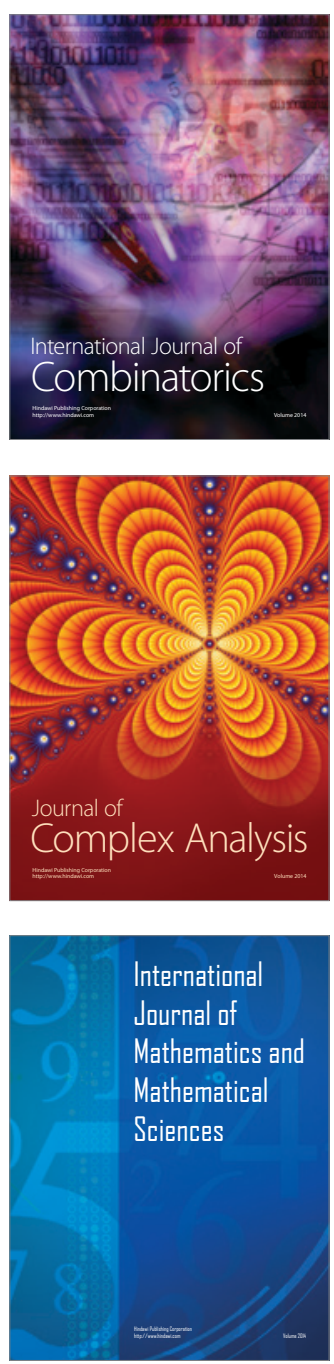
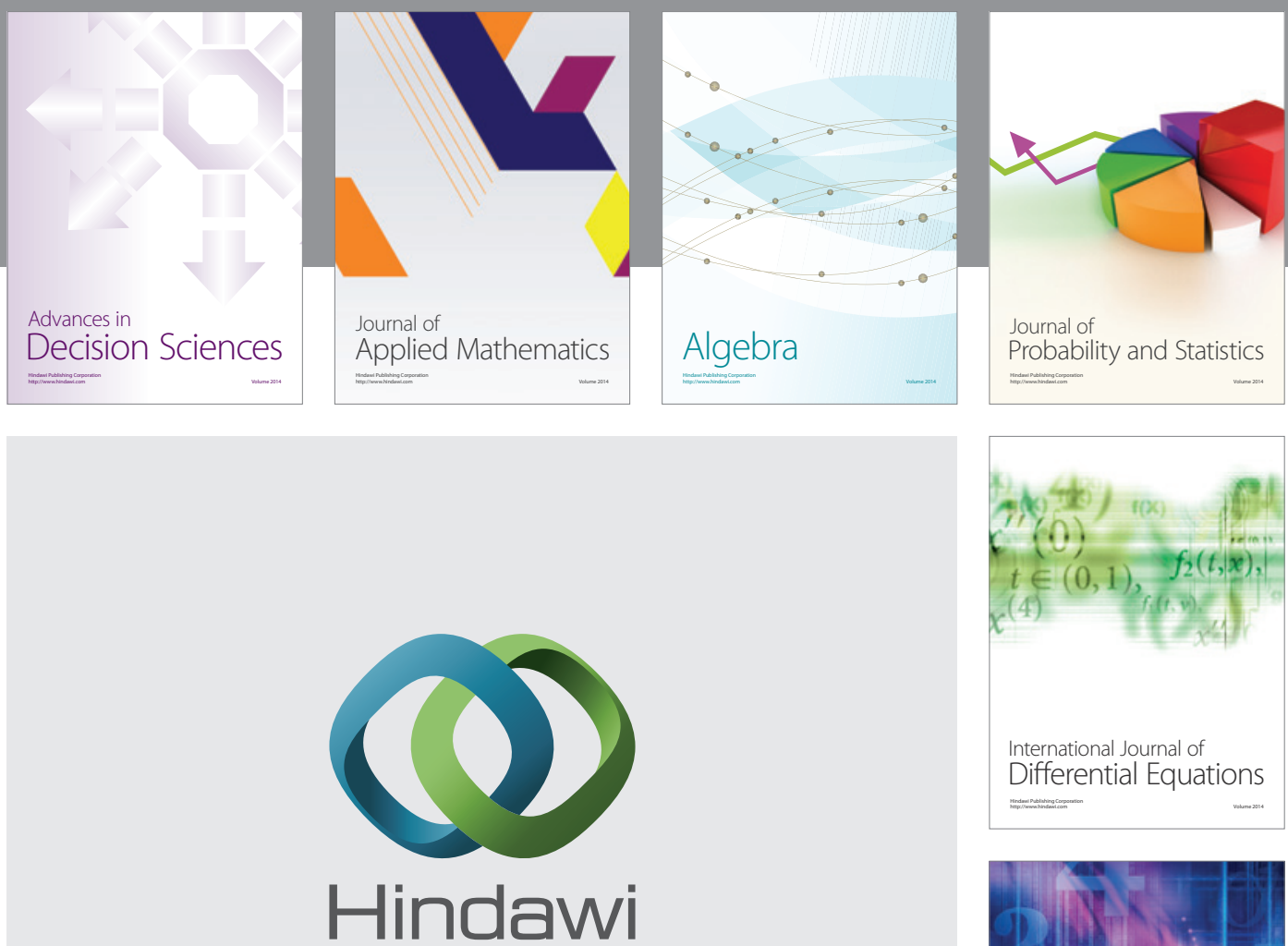

Submit your manuscripts at http://www.hindawi.com
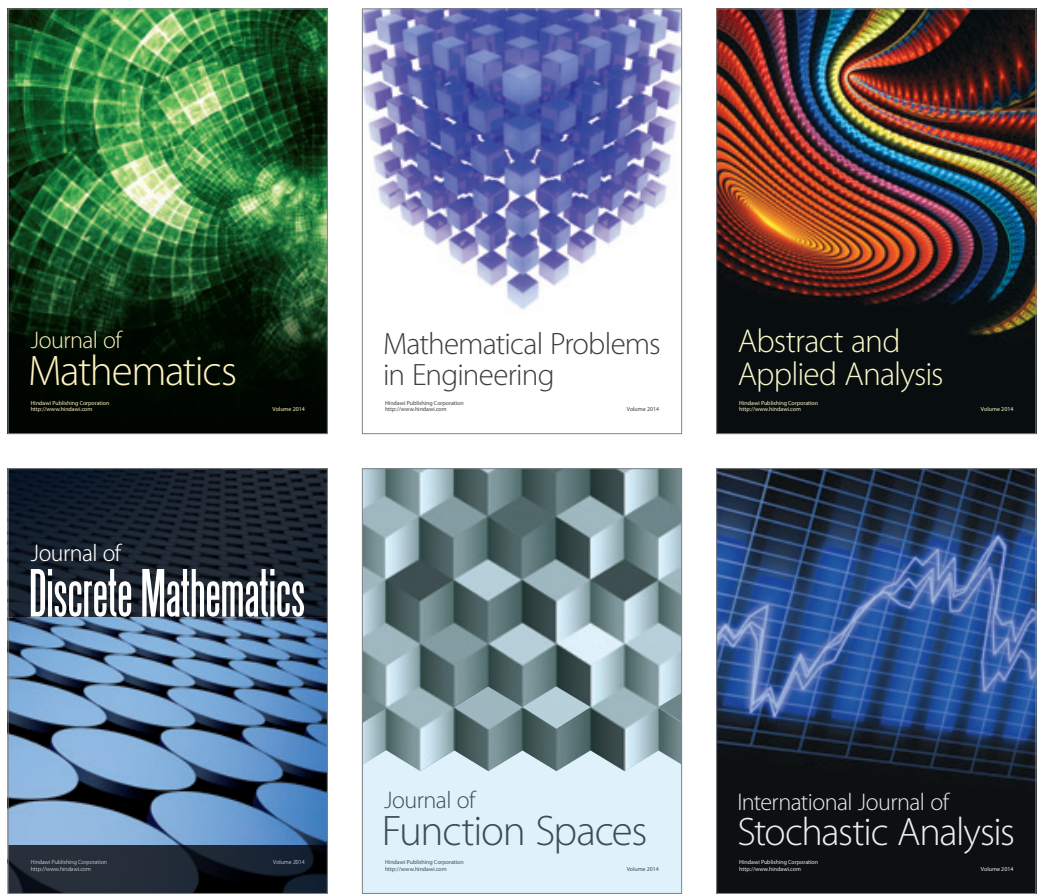

Journal of

Function Spaces

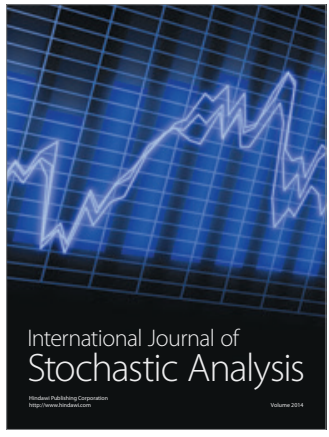

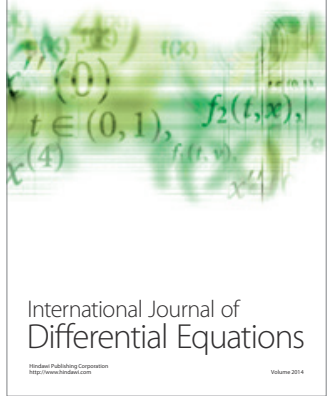
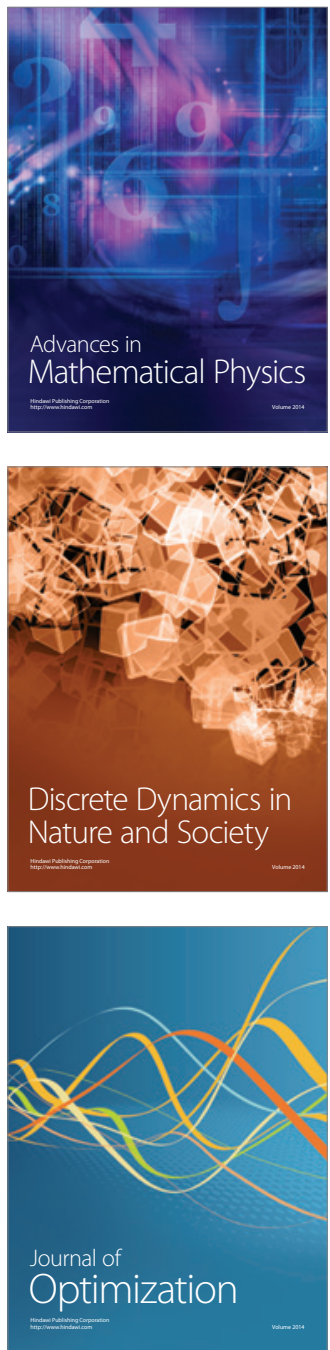